\title{
NONLINEAR PERISTALTIC TRANSPORT OF MHD FLOW THROUGH A POROUS MEDIUM
}

\author{
KH. S. MEKHEIMER and T. H. AL-ARABI
}

Received 28 May 2001

\begin{abstract}
In order to determine the characteristics of peristaltic transport of magnetohydrodynamic flow through a porous medium, the motion of a hydromagnetic (electrically conducting), viscous, and incompressible fluid in planer channel filled with a homogeneous porous medium and having electrically insulated walls that are transversely displaced by an infinite, harmonic travelling wave of large wavelength was analyzed using a perturbation expansion in terms of a variant wave number. We obtain an explicit form for the velocity field, a relation between the pressure rise and flow rate, in terms of Reynolds number, wave number, Hartmann number, permeability parameter, and the occlusion. The effects of all parameters of the problem are numerically discussed and graphically explained.
\end{abstract}

2000 Mathematics Subject Classification: 76S05.

1. Introduction. Peristalsis is now well known to the physiologists as one of the major mechanisms for fluid transport in many biological systems. In particular, peristaltic mechanism may be involved in swallowing food through the oesophagus, urine transport from kidney to bladder through the ureter, movement of chyme in the gastrointestinal tract, transport of spermatozoa in the ductus efferents of the male reproductive tracts and in the cervical canal, movement of the ovum in the fallopian tubes, and in the vasomotion of small blood vessels as well as blood flow in arteries. In addition, peristaltic pumping occurs in many practical applications involving biomechanical systems. Also, finger and roller pumps are frequently used for pumps corrosive or very pure materials so as to prevent direct contact of the fluid with the pump's internal surfaces. A number of analytical [3, 5, 7, 9, 11, 12, 16, 23], numerical, and experimental $[2,10,19,20,21]$ studies of peristaltic flow of different fluids have been reported. Several review articles have been written [8, 14]. Also a summary of analytical papers up to 1984 has been presented in [18]. Most of the analytical studies use perturbation series in a small parameter such as Reynolds number or a dimensionless wave number, which, unfortunately, limits the range of validity of the results. However, a perturbation method does provide explicit information about the physical effects of that parameter. Also, the analytical results can be used to check the calculations of wider-range numerical methods. 
It has been established that the biological systems, in general, are greatly addicted by the application of the external magnetic field. Moreover, the MHD flow of a fluid in a channel with elastic rhythmically contracting walls (peristaltic flow) is of interest in connection with certain problems of the movement of conductive physiological fluids, and with the need for theoretical research on the operation of a peristaltic MHD compressor, also the principle of magnetic field may be used in clinical application (magnetic resonance imaging MRI). The effect of moving magnetic field on blood flow was studied by Agrawal and Anwaruddin [1], they observed, for the flow of blood in arteries with arterial disease like arterial stenosis or arteriosclerosis, that the influence of magnetic field may be utilized as a blood pump in carrying out cardiac operations. Also, the magnetohydrodynamic flow with suspension has been studied by Parsad and Ramacharyulu [6], and Srivastava and Agrawal [17] considered the blood as an electrically conducting fluid and that it constitutes a suspension of red cells in plasma.

Flow through a porous medium has been studied by a number of workers employing Darcy's law [15]. Some studies about this point have been made by Varshney [22], Raptis and Perdikis [13], and El-Dabe and El-Mohendis [4].

Here, we are interested in the nonlinear peristaltic pumping of MHD flow through a porous medium, and due to the complexity of the nonlinear equations of motion, we only consider the case: a symmetric, harmonic, infinite wave train having a wavelength that is large relative to the gap between the walls; transverse displacement only; and electrically conducting fluid. This problem may be considered as a mathematical representation to the case of gall bladder and bile duct with stones under a uniform magnetic field. The gall stones cause fibrosis of the gall bladder, thus when a stone is later impacted in the common bile duct, jaundice results and gall bladder cannot dilate as it fibrosed as a result of the cholecystitis due to stones.

A regular perturbation series is used to solve the problem; variables are expanded in a power series of the parameter $\delta$, which is defined as the ratio of half width of the channel to the wavelength of the peristaltic wave. Closed form solutions up to order $\delta^{2}$ are presented.

2. Formulation of the problem. Consider the two-dimensional unsteady hydromagnetic flow of a viscous, incompressible, and electrically conducting fluid in an infinite channel having width $2 a$ and filled with a homogeneous porous medium. A uniform magnetic filed $B_{0}^{\prime}$ is acting along the $\bar{Y}$-axis and the induced magnetic field is assumed to be negligible. We assume an infinite wave train travelling with velocity $c$ along an electrically insulated walls. We choose a rectangular coordinate system for the channel with $\bar{X}$ along the center line in the direction of wave propagation, and $\bar{Y}$ transverse to it. The geometry of the wall surface is defined as 


$$
\bar{h}(\bar{X}, t)=a+b \sin \left\{\frac{2 \pi}{\lambda}(\bar{X}-c t)\right\},
$$

where $b$ is the wave amplitude and $\lambda$ is the wavelength.

We carry out this investigation in a coordinate system moving with the wave speed, in which the boundary shape is stationary. The coordinates and velocities in the laboratory frame $(\bar{X}, \bar{Y})$ and the wave frame $(\bar{x}, \bar{y})$ are related by

$$
\begin{array}{cl}
\bar{x}=\bar{X}-c t, & \bar{y}=\bar{Y}, \\
\bar{u}=\bar{U}-c, & \bar{v}=\bar{V},
\end{array}
$$

where $\bar{U}, \bar{V}$ and $\bar{u}, \bar{v}$ are the velocities components in the corresponding coordinate systems.

Using these transformations and introducing the following dimensionless variables:

$$
\begin{gathered}
x=\frac{2 \pi \bar{x}}{\lambda}, \quad y=\frac{\bar{y}}{a}, \quad u=\frac{\bar{u}}{c}, \quad v=\frac{\bar{v}}{c}, \\
h=\frac{h(\bar{x})}{a}, \quad p=\frac{2 \pi a^{2}}{\lambda \mu c} \bar{p}(\bar{x}), \quad \tau=\frac{2 \pi c t}{\lambda}, \quad \psi=\frac{\bar{\psi}}{c a},
\end{gathered}
$$

we find that the equation which governs the MHD flow in terms of the stream function $\psi(x, y)$ after eliminating the pressure gradient is

$$
\delta \operatorname{Re}\left\{\left(\frac{\partial \psi}{\partial y} \frac{\partial}{\partial x}-\frac{\partial \psi}{\partial x} \frac{\partial}{\partial y}\right) \nabla^{2} \psi\right\}=\nabla^{4} \psi-M^{2} \frac{\partial^{2} \psi}{\partial y^{2}}-\frac{1}{K} \nabla^{2} \psi
$$

where

$$
u=\frac{\partial \psi}{\partial y}, \quad v=-\delta \frac{\partial \psi}{\partial x}, \quad \nabla^{2}=\delta^{2} \frac{\partial^{2}}{\partial x^{2}}+\frac{\partial^{2}}{\partial y^{2}}
$$

and the dimensionless parameters: Reynolds number $\mathrm{Re}=c a \rho / \mu$, wave number $\delta=2 \pi a / \lambda$, Hartmann number $M=\sqrt{\sigma / \mu} B_{o} a$ (suitably greater than $\sqrt{2}$ ), and permeability parameter $K=k / a^{2}$, where $\rho$ is the density, $\mu$ the viscosity of the fluid, $\sigma$ electrical conductivity of the fluid, and $k$ the permeability of the porous medium.

3. Rate of volume flow and boundary conditions. The instantaneous volume flow rate in the fixed frame is given by

$$
Q=\int_{0}^{\bar{h}} \bar{U}(\bar{X}, \bar{Y}, t) d \bar{Y}
$$

where $\bar{h}$ is a function of $\bar{X}$ and $t$. 
The rate of volume flow in the wave frame is given by

$$
q=\int_{0}^{\bar{h}} \bar{u}(\bar{x}, \bar{y}) d \bar{y}
$$

where $\bar{h}$ is a function of $\bar{x}$ alone. If we substitute (2.2) into (3.1) and make use of (3.2), we find that the two rates of volume flow are related through

$$
Q=q+c \bar{h}
$$

The time-mean flow over a period $T$ at a fixed position $\bar{X}$ is defined as

$$
\bar{Q}=\frac{1}{T} \int_{0}^{T} Q d t
$$

Substituting (3.3) into (3.4) and integrating, we get

$$
\bar{Q}=q+a c
$$

On defining the dimensionless time-mean flows $\theta$ and $F$, respectively, in the fixed and wave frame as

$$
\theta=\frac{\bar{Q}}{a c}, \quad F=\frac{q}{a c},
$$

one finds that (3.5) may be written as

$$
\theta=F+1
$$

where

$$
F=\int_{0}^{h} \frac{\partial \psi}{\partial y} d y=\psi(h)-\psi(0)
$$

We note that $h$ represents the dimensionless form of the surface of the peristaltic wall:

$$
h(x)=1+\phi \sin x
$$

where

$$
\phi=\frac{b}{a}
$$

is the amplitude ratio or the occlusion.

If we select the zero value of the streamline at the streamline $(y=0)$ :

$$
\psi(0)=0,
$$


then the wall $(y=h)$ is a streamline of value

$$
\psi(h)=F
$$

The boundary conditions for the dimensionless stream function in the wave frame are

$$
\begin{gathered}
\psi=0, \quad \frac{\partial^{2} \psi}{\partial y^{2}}=0, \quad \text { on } y=0, \\
\frac{\partial \psi}{\partial y}=-1, \quad \psi=F, \quad \text { on } y=h .
\end{gathered}
$$

4. Perturbation solution. In order to solve the present problem, we expand the flow quantities in a power series of the small parameter $\delta$ as follows:

$$
\begin{aligned}
\psi & =\psi_{0}+\delta \psi_{1}+\delta^{2} \psi_{2}+\cdots, \\
F & =F_{0}+\delta F_{1}+\delta^{2} F_{2}+\cdots \\
\frac{\partial p}{\partial x} & =\frac{\partial p_{0}}{\partial x}+\delta \frac{\partial p_{1}}{\partial x}+\delta^{2} \frac{\partial p_{2}}{\partial x}+\cdots
\end{aligned}
$$

On substituting (4.1) into (2.4) and (3.13), collecting terms of equal powers of $\delta$, and then equating the coefficients of like powers on both sides of the equations, we obtain the following set of problems.

4.1. Zero-order problem. We need to solve

$$
\left(\frac{\partial^{2}}{\partial y^{2}}-\gamma^{2}\right) \frac{\partial^{2} \psi_{0}}{\partial y^{2}}=0
$$

subject to the boundary conditions:

$$
\begin{gathered}
\psi_{0}=0, \quad \frac{\partial^{2} \psi_{0}}{\partial y^{2}}=0, \quad \text { on } y=0, \\
\psi_{0}=F_{0}, \quad \frac{\partial \psi_{0}}{\partial y}=-1, \quad \text { on } y=h
\end{gathered}
$$

where $\gamma=\sqrt{1 / K+M^{2}}$.

The solution of (4.2) in terms of stream function is given by

$$
\psi_{0}=\left(\frac{F_{0} \gamma+\tanh \gamma h}{\gamma h-\tanh \gamma h}\right)\left(y-\frac{\sinh \gamma y}{\gamma \cosh \gamma h}\right)-\frac{\sinh \gamma y}{\gamma \cosh \gamma h} .
$$

We point out that this problem is essentially the classical Poiseuille MHD flow. It can be easily shown that [9]

$$
\lim _{\gamma \rightarrow 0} \psi_{0}=-\frac{3}{2}\left(F_{0}+h\right)\left\{\frac{1}{3} \frac{y^{3}}{h^{3}}-\frac{y}{h}\right\}-y .
$$


4.2. First-order problem. On substituting the zero-order solution (4.4) into the equation of motion obtained for first order in $\delta$, one finds that the latter equation reduces to

$$
\left(\frac{\partial^{2}}{\partial y^{2}}-\gamma^{2}\right) \frac{\partial^{2} \psi_{1}}{\partial y^{2}}=\operatorname{Re}\left\{\left(\frac{\partial \psi_{0}}{\partial y} \frac{\partial}{\partial x}-\frac{\partial \psi_{0}}{\partial x} \frac{\partial}{\partial y}\right) \frac{\partial^{2} \psi_{0}}{\partial y^{2}}\right\}
$$

The solution of (4.6) subject to the first-order boundary conditions:

$$
\begin{aligned}
& \psi_{1}=0, \quad \frac{\partial^{2} \psi_{1}}{\partial y^{2}}=0, \quad \text { on } y=0 \text {, } \\
& \psi_{1}=F_{1}, \quad \frac{\partial \psi_{1}}{\partial y}=0, \quad \text { on } y=h \text {, }
\end{aligned}
$$

is given as

$$
\begin{aligned}
\psi_{1}= & c_{11} y^{2} \sinh \gamma y+c_{12} y \cosh \gamma y+c_{13} \sinh \gamma y \\
& +\frac{F_{1}(\sinh \gamma y-\gamma y \cosh \gamma y)}{\sinh \gamma h-\gamma h \cosh \gamma h}+D y,
\end{aligned}
$$

where the constants $c_{11}, c_{12}, c_{13}$, and $D$ are listed in Appendix A.

4.3. Second-order problem. As before, we insert the zero- and first-order solutions into the equations of motion obtained for $\delta^{2}$ and find that

$$
\begin{aligned}
\left(\frac{\partial^{2}}{\partial y^{2}}-\gamma^{2}\right) \frac{\partial^{2} \psi_{2}}{\partial y^{2}}=\operatorname{Re}\{ & \left(\frac{\partial \psi_{1}}{\partial y} \frac{\partial}{\partial x}-\frac{\partial \psi_{1}}{\partial x} \frac{\partial}{\partial y}\right) \frac{\partial^{2} \psi_{0}}{\partial y^{2}} \\
+ & \left.\left(\frac{\partial \psi_{0}}{\partial y} \frac{\partial}{\partial x}-\frac{\partial \psi_{0}}{\partial x} \frac{\partial}{\partial y}\right) \frac{\partial^{2} \psi_{1}}{\partial y^{2}}\right\}-2 \frac{\partial^{4} \psi_{0}}{\partial y^{2} \partial x^{2}}+\frac{1}{K} \frac{\partial^{2} \psi_{0}}{\partial x^{2}}
\end{aligned}
$$

Using the zero-order and the first-order solutions in (4.9) and then applying the boundary conditions:

$$
\begin{aligned}
& \psi_{2}=0, \quad \frac{\partial^{2} \psi_{2}}{\partial y^{2}}=0, \quad \text { on } y=0, \\
& \psi_{2}=F_{2}, \quad \frac{\partial \psi_{2}}{\partial y}=0, \quad \text { on } y=h,
\end{aligned}
$$

we find that the stream function $\psi_{2}$ turns out to be

$$
\begin{aligned}
\psi_{2}= & c_{34} y^{4} \sinh \gamma y+c_{35} y^{3} \cosh \gamma y+c_{36} y^{2} \sinh \gamma y \\
& +c_{37} y \cosh \gamma y+c_{38} \sinh \gamma y+c_{39} y \cosh 2 \gamma y+c_{40} \sinh 2 \gamma y \\
& -\frac{c_{29}}{\gamma^{2}}\left(y^{3}+\frac{6 y}{\gamma^{2}}\right)-\frac{A}{\gamma^{2}} y+\frac{F_{2}(\sinh \gamma y-\gamma y \cosh \gamma y)}{\sinh \gamma h-\gamma h \cosh \gamma h}+E \sinh \gamma y .
\end{aligned}
$$


Here, the coefficients $c_{34}, c_{35}, c_{36}, c_{37}, c_{38}, c_{39}, c_{40}, c_{29}, A$, and $E$ are listed in Appendix B.

Summing up, we write the perturbation solution through order 2 as follows:

$$
\begin{aligned}
\psi=\left(\frac{F_{0} \gamma+\tanh \gamma h}{h \gamma-\tanh \gamma h}\right)\left(y-\frac{\sinh \gamma y}{\gamma \cosh \gamma h}\right)-\frac{\sinh \gamma y}{\gamma \cosh \gamma h} \\
+\delta\left\{c_{11} y^{2} \sinh \gamma y+c_{12} y \cosh \gamma y+c_{13} \sinh \gamma y\right. \\
\left.+\frac{\left(F_{1} \sinh \gamma y-\gamma y \cosh \gamma y\right)}{\sinh \gamma h-\gamma h \cosh \gamma h}+D y\right\} \\
+\delta^{2}\left\{c_{34} y^{4} \sinh \gamma y+c_{35} y^{3} \cosh \gamma y+c_{36} y^{2} \sinh \gamma y\right. \\
+c_{37} y \cosh \gamma y+c_{38} \sinh \gamma y+c_{39} y \cosh 2 \gamma y+c_{40} \sinh 2 \gamma y \\
\quad-\left(\frac{c_{29}}{\gamma^{2}}\right)\left(y^{3}+\frac{6 y}{\gamma^{2}}\right)-\left(\frac{A}{\gamma^{2}}\right) y \\
\left.+\frac{F_{2}(\sinh \gamma y-\gamma y \cosh \gamma y)}{\sinh \gamma h-\gamma h \cosh \gamma h}+E \sinh \gamma y\right\} .
\end{aligned}
$$

5. Pressure gradient. When the flow is steady in the wave frame, one can characterize the pumping performance by means of the pressure rise per wavelength. On substituting (4.1) into the dimensionless equation of motion and equating the coefficient of like powers of $\delta$ on both sides of the equation, we obtain a set of partial differential equations for $\partial p_{0} / \partial x, \partial p_{1} / \partial x$, and $\partial p_{2} / \partial x$.

The nondimensional pressure rise and the nondimensional friction force per wavelength in the wave frame are defined, respectively, as

$$
\triangle p_{\lambda}=\frac{2 \pi a^{2}}{\lambda \mu c} \triangle \bar{p}_{\lambda}=\int_{0}^{2 \pi}\left(\frac{d p}{d x}\right) d x, \quad F_{\lambda}=\int_{0}^{2 \pi}-h\left(\frac{d p}{d x}\right) d x
$$

Since $\partial p / \partial x$ is periodic in $x$, the pressure rise and the friction force per wavelength in the longitudinal direction are independent of $y$ [11], and the integrals in (5.1) can be evaluated numerically on the axis at $y=0$. Now,

$$
\begin{aligned}
\triangle p_{\lambda} & =\triangle p_{\lambda^{0}}+\delta \triangle p_{\lambda^{1}}+\delta^{2} \triangle p_{\lambda^{2}}+\cdots, \\
F_{\lambda} & =F_{\lambda^{0}}+\delta F_{\lambda^{1}}+\delta^{2} F_{\lambda^{2}}+\cdots,
\end{aligned}
$$

and we compute the pressure rise and friction force per wavelength at each order for a wall shape of the sinusoidal form defined by (3.9). Using the zero-, 
first-, and second-order terms for the pressure gradient in (5.1), we obtain

$$
\begin{aligned}
& \Delta p_{\lambda}^{(2)}=\int_{0}^{2 \pi}\left\{-\frac{\gamma^{2}\left(F^{(2)}+\gamma^{-1} \tanh \gamma h\right)}{\left(h-\gamma^{-1} \tanh \gamma h\right)}\right. \\
& +\delta\left[-\frac{\gamma^{2}\left(c_{14}-h c_{15}\right)}{\left(h-\gamma^{-1} \tanh \gamma h\right)}-\gamma^{2} c_{15}-\operatorname{Re}\left(c_{01} c_{01}^{\prime}+c_{02} c_{02}^{\prime}\right)\right] \\
& +\delta^{2}\left[6 c_{35}+6 \gamma c_{36}+2 \gamma^{2} c_{37}+11 \gamma^{2} c_{39}+6 \gamma^{3} c_{40}+A+\left(c_{01}^{\prime \prime}-c_{02}^{\prime \prime}\right)\right. \\
& -\operatorname{Re}\left[\left(c_{12}-\gamma^{2} D+\gamma c_{13}\right)\left(c_{01}^{\prime}-c_{02}^{\prime}\right)\right. \\
& \left.\left.\left.+\left(c_{01}-c_{02}\right)\left(c_{12}^{\prime}-\gamma^{-2} D^{\prime}+\gamma c_{13}^{\prime}\right)\right]\right]\right\} d x, \\
& F_{\lambda}^{(2)}=\int_{0}^{2 \pi}\left\{\frac{h \gamma^{2}\left(F^{(2)}+\gamma^{-1} \tanh \gamma h\right)}{\left(h-\gamma^{-1} \tanh \gamma h\right)}\right. \\
& +h \delta\left[\frac{\gamma^{2}\left(c_{14}-h c_{15}\right)}{\left(h-\gamma^{-1} \tanh \gamma h\right)}+\gamma^{2} c_{15}+\operatorname{Re}\left(c_{01} c_{01}^{\prime}+c_{02} c_{02}^{\prime}\right)\right] \\
& -h \delta^{2}\left[6 c_{35}+6 \gamma c_{36}+2 \gamma^{2} c_{37}+11 \gamma^{2} c_{39}+6 \gamma^{3} c_{40}+A+\left(c_{01}^{\prime \prime}-c_{02}^{\prime \prime}\right)\right. \\
& -\operatorname{Re}\left[\left(c_{12}-\gamma^{-2} D+\gamma c_{13}\right)\left(c_{01}^{\prime}-c_{02}^{\prime}\right)\right. \\
& \left.\left.\left.+\left(c_{01}-c_{02}\right)\left(c_{12}^{\prime}-\gamma^{-2} D^{\prime}+\gamma c_{13}^{\prime}\right)\right]\right]\right\} d x,
\end{aligned}
$$

where $\triangle p_{\lambda}^{(2)}, F_{\lambda}^{(2)}$, and $F^{(2)}$ are the pressure rise, the friction force, and the flow rate, respectively, in the wave frame to the second order in $\delta$,

$$
\begin{aligned}
\Delta p_{\lambda}^{(2)} & \equiv \Delta p_{\lambda^{0}}+\delta \Delta p_{\lambda^{1}}+\delta^{2} \Delta p_{\lambda^{2}}, \\
F_{\lambda}^{(2)} & \equiv F_{\lambda^{0}}+\delta F_{\lambda^{1}}+\delta^{2} F_{\lambda^{2}}, \\
F^{(2)} & \equiv F_{0}+\delta F_{1}+\delta^{2} F_{2} .
\end{aligned}
$$

We note that the relation between the dimensionless flow rate in the frame $F^{(2)}$ and that in the time-mean flow rate in the lab frame $\theta^{(2)}$ is given by

$$
\theta^{(2)}=F^{(2)}+1
$$

We have here the second order of the pressure rise as a nonlinear function of the time-mean flow. For completeness, we give the second-order expression 
of the stream function $\psi^{(2)}$ in terms of $F^{(2)}$,

$$
\begin{aligned}
\psi^{(2)}= & \frac{\left(F^{(2)}+(1 / \gamma) \tanh \gamma h\right) y}{h-(1 / \gamma) \tanh \gamma h}-\frac{\left(F^{(2)}+h\right) \sinh \gamma y}{\gamma \cosh \gamma h(h-(1 / \gamma) \tanh \gamma h)} \\
+ & \delta\left\{c_{11} y^{2} \sinh \gamma y+c_{12} y \cosh \gamma y+c_{13} \sinh \gamma y+D y\right\} \\
+ & \delta^{2}\left\{c_{34} y^{4} \sinh \gamma y+c_{35} y^{3} \cosh \gamma y+c_{36} y^{2} \sinh \gamma y\right. \\
& +c_{37} y \cosh \gamma y+c_{38} \sinh \gamma y+c_{39} y \cosh 2 \gamma y \\
& \left.+c_{40} \sinh 2 \gamma y-\frac{c_{29}}{\gamma^{2}}\left(y^{3}+\frac{6 y}{\gamma^{2}}\right)-\frac{A}{\gamma^{2}} y+E \sinh \gamma y\right\} .
\end{aligned}
$$

6. Discussion of results. The results of our analysis are presented as follows:

(1) pumping characteristics;

(2) the streamlines and trapping regions for the parameters $\{\operatorname{Re}, \varphi, \delta, \gamma$, $\left.\theta^{(2)}\right\}$.

6.1. Pumping characteristics. Figure 6.1 is a graph of the dimensionless pressure change per wavelength $\left(\triangle p_{\lambda}^{(2)}\right)$ versus the dimensionless flow rate $\left(\theta^{(2)}\right)$ for the case $\{\operatorname{Re}=0, \phi=0.5, M=2.5$ (MHD), $K=0.8, \delta=0,1,2,3\}$. The graph is sectored so that the upper right-hand quadrant (I) denotes the region of peristaltic pumping, where $\theta^{(2)}>0$ (positive pumping) and $\Delta p_{\lambda}^{(2)}>0$ (adverse pressure gradient). Quadrant (II), where $\Delta p_{\lambda}^{(2)}<0$ (favorable pressure gradient) and $\theta^{(2)}>0$ (positive pumping), is designated as augmented flow. Quadrant (IV) such that $\triangle p_{\lambda}^{(2)}>0$ (adverse pressure gradient) and $\theta^{(2)}<0$ is called retrograde or backward pumping. The flow is opposite to the direction of the peristaltic motion.

Figure 6.1(a) shows that the peristaltic pumping rate $\left(\theta^{(2)}\right)$ increases (for the same $\left.\Delta p_{\lambda}^{(2)}\right)$ as $\delta(\equiv 2 \pi a / \lambda)$ increases. Also shown in Figure 6.1(a) the case for $\varphi=0, \gamma \rightarrow 0(M \rightarrow 0$ and $K \rightarrow \infty)$ which is the classical Poiseuille flow through a two-dimensional channel. Figure 6.1(b) is similar to Figure 6.1(a) except that $\mathrm{Re}=10$ and $\delta=0,0.05,0.1,0.15$. It is clear that the relation between $\triangle p_{\lambda}^{(2)}$ (pressure change per wavelength) and the flow rate $\theta^{(2)}$ is linear for $\operatorname{Re}=0$ and nonlinear for $\mathrm{Re} \neq 0$.

Figure 6.2(a) is a graph of the pressure change per wavelength $\left(\triangle p_{\lambda}^{(2)}\right)$ versus the observer flow rate $\left(\theta^{(2)}\right)$ for $\{\varphi=0.3, \operatorname{Re}=1, \delta=0.06, K=0.8, M=$ $2,4,6,8\}$. Figure $6 \cdot 2(\mathrm{~b})$ is a graph of the dimensionless friction force versus the observer flow rate for $\{\varphi=0.3, \operatorname{Re}=1, \delta=0.06, K=0.8, M=2,4,6,8\}$. We observe that an increase in $M$ results in an increase in the peristaltic pumping rate (for the same $\left(\triangle p_{\lambda}^{(2)}\right)$ ) and also in an increase in the pressure rise. Also we observe that the friction force has the opposite behavior compared to the pressure rise. 


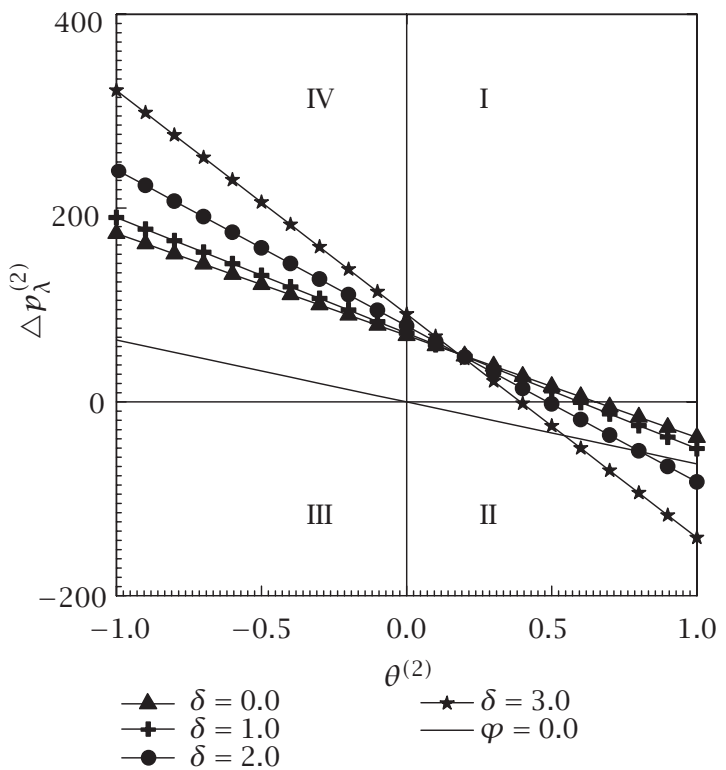

(a)

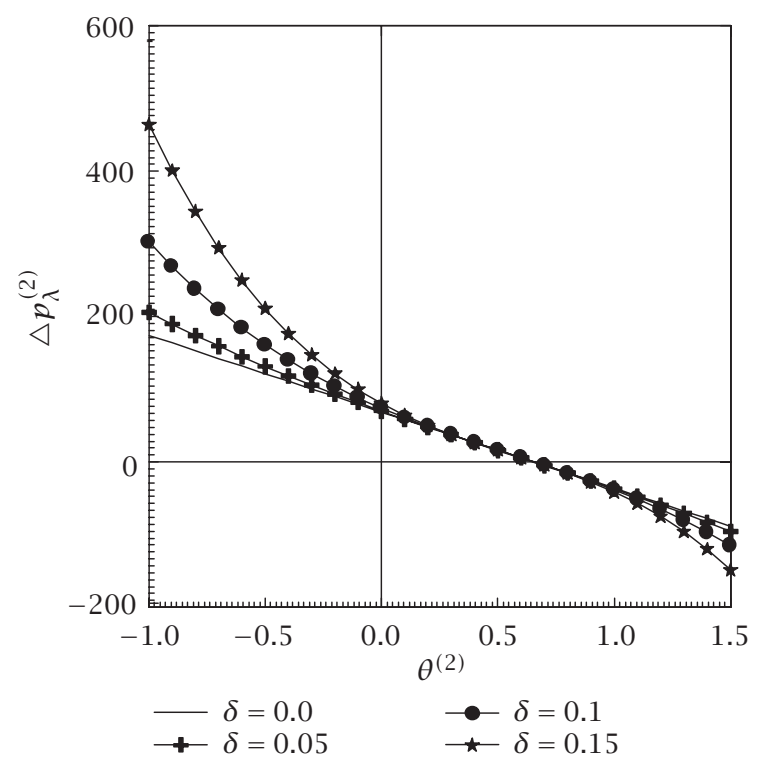

(b)

FIGURE 6.1. The pressure gradient per wavelength $\triangle p_{\lambda}^{(2)}$ versus the dimensionless flow rate $\theta^{(2)}$ for $\varphi=0.5, M=2.5, K=0.8$, and different values of $\delta$, at (a) $\operatorname{Re}=0$ and (b) $\operatorname{Re}=10(\varphi \rightarrow 0, \gamma \rightarrow 0$ Poiseuille flow). 


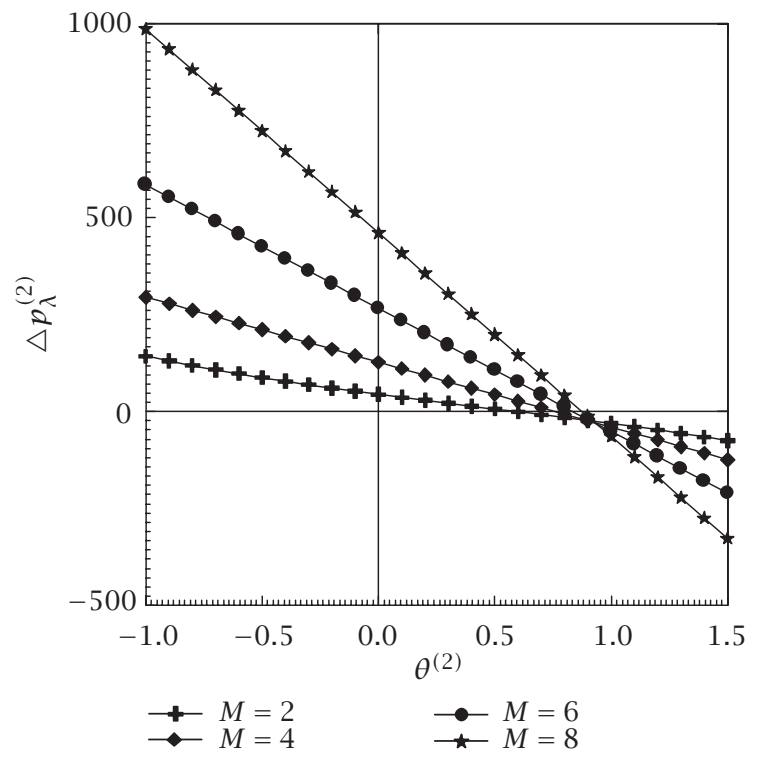

(a) The pressure gradient per wavelength $\triangle p_{\lambda}^{(2)}$ versus the dimensionless flow rate $\theta^{(2)}$ for $\operatorname{Re}=1, \varphi=0.3, K=0.8$, $\delta=0.06$, and different values of $M$.

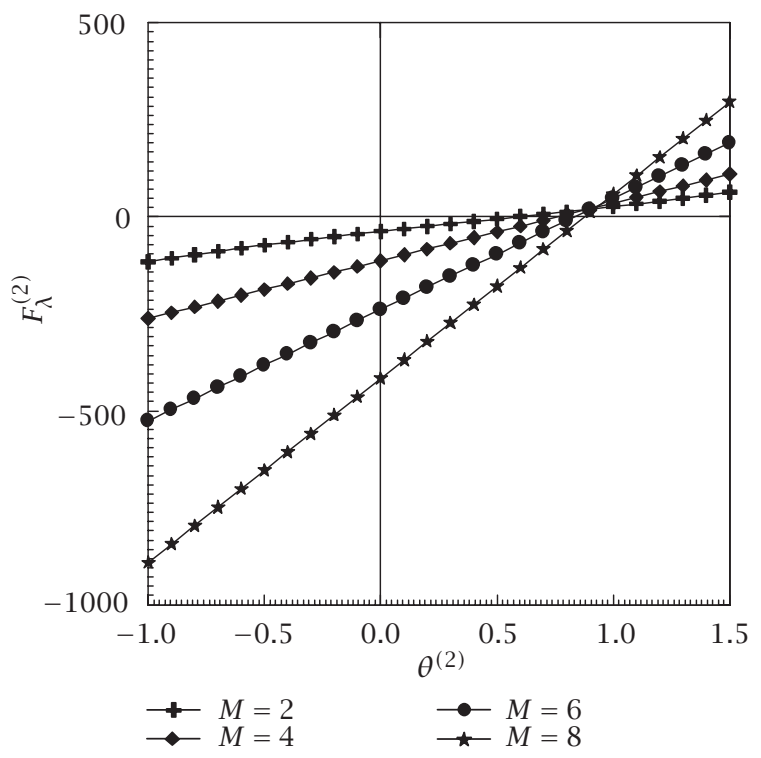

(b) The frictionless force per wavelength $F_{\lambda}^{(2)}$ versus the dimensionless flow rate $\theta^{(2)}$ for $\operatorname{Re}=1, \varphi=0.3, K=0.8$, $\delta=0.06$, and different values of $M$. 
Figure 6.3(a) is a graph of the pressure change per wavelength $\left(\triangle p_{\lambda}^{(2)}\right)$ versus the observer flow rate $\left(\theta^{(2)}\right)$ for $\{\varphi=0.3, \delta=0.06, M=2, \operatorname{Re}=1$, $K=0.2,0.4,1,3\}$. Figure $6.3(\mathrm{~b})$ is a graph of the dimensionless friction force versus the observer flow rate for $\{\varphi=0.3, \delta=0.06, M=2, \operatorname{Re}=1, K=0.2,0.4,1,3\}$. We observe that an increase in $K$ results in a decrease in the peristaltic pumping rate (for the same $\left.\left(\triangle p_{\lambda}^{(2)}\right)\right)$, and also, a decrease in the pressure rise. Also the friction force has the opposite behavior compared to the pressure rise.

We define the maximum pressure rise $\left(\triangle p_{\lambda}^{(2)}\right)_{\max }$, which is obtained by putting $\theta^{(2)}=0$ and would occur in a channel, whose exit was shut off, as $\left(\triangle p_{\theta=0}^{(2)}\right)$. Figure 6.4(a) is a graph of $\left(\triangle p_{\lambda}^{(2)}\right)_{\max }$ versus $K$ for varying values of $M$ at $\delta=0.06, \varphi=0.2$, and $\mathrm{Re}=1$, which shows the effects of $M$ and $K$ on the pumping rate, and we observe that for the same $\left(\triangle p_{\lambda}^{(2)}\right)_{\max }$ the affect of magnetic field increases as permeability parameter increases. Figure $6.4(\mathrm{~b})$ is a graph of the maximum pressure rise $\left(\triangle p_{\lambda}^{(2)}\right)_{\max }$ versus the amplitude ratio $\varphi$ for different values of $K$ at $\delta=0.06, M=2.5$, and $\mathrm{Re}=1$. It is clear that the maximum pressure rise increases as $\varphi$ and $M$ increase and decrease as $K$ increases.

6.2. Streamlines and fluid trapping. The phenomenon of trapping, whereby a bolus (defined as a volume of fluid bounded by a closed streamlines in the wave frame) is transported at the wave speed, has been studied by several investigators (Shapiro et al. [9], Jaffrin [7], and Siddiqui and Schwarz [16]). Figures 6.5(a), 6.5(b), and 6.5(c) are graphs of the streamlines for the conditions $\{\varphi=0.4, \delta=0.06, M=1.5, K=1, \operatorname{Re}=0\}$, and $\theta^{(2)}=0.4,1.2,1.8$, respectively. In Figure 6.5(a) the case of typical Stokes nontrapping region for peristaltic pumping, the adverse pressure gradient $\left(\triangle p_{\lambda}^{(2)}\right)>0$ is almost large enough to cause zero pumping. While Figure 6.5(b) shows the center line trapped eddy, which was described by Jaffrin [7] and Siddiqui [16], the width of the eddy (measured on the centerline) increases and the eddy lifts off the center line and forms a torus-shaped eddy with flow through the center, in case of increasing $\theta^{(2)}$. We also observe that a smaller trapped bolus in the crest of the peristaltic wave (see Figure 6.5(c)), and as $\theta^{2}$ becomes large, the flow has the same form as the flow through a two-dimensional symmetric channel, having an axial sinusoidal variation of its width.

The effect of the Hartmann number $M$ on the peristaltic velocity is demonstrated in Figures 6.6(a), 6.6(b), and 6.6(c) for $\left\{\varphi=0.4, \delta=0.06, \operatorname{Re}=1, \theta^{(2)}=\right.$ $0.8, K=1, M=2,5,7\}$, which shows that as the $M$ increases the center line trapped eddy disappears and the fluid moves like a block, and as $M$ becomes larger the rigidity of the fluid appears. The effect of the permeability parameter on the peristaltic velocity is demonstrated in Figures 6.7(a), 6.7(b), and 6.7(c) for $\left\{\varphi=0.4, \delta=0.06\right.$, $\left.\operatorname{Re}=1.0, \theta^{(2)}=0.8, M=2, K=0.02,0.08,0.15\right\}$, which shows that as the permeability parameter increases the center line trapped eddy appears. Small values of $K$ shows some sort of rigidity of fluid. 


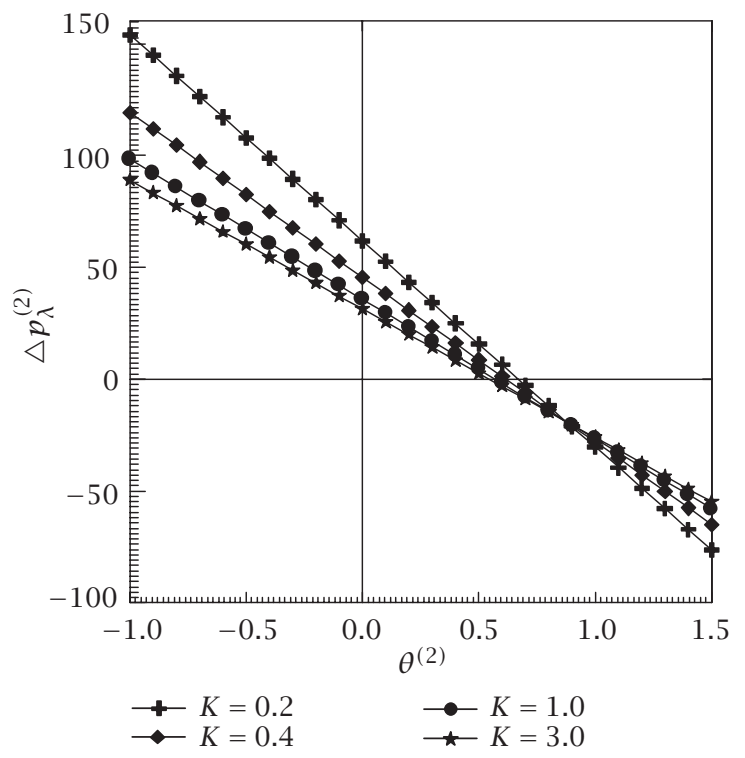

(a) The pressure gradient per wavelength $\triangle p_{\lambda}^{(2)}$ versus the dimensionless flow rate $\theta^{(2)}$ for $\operatorname{Re}=1, \varphi=0.3, M=2$, $\delta=0.06$, and different values of $K$.

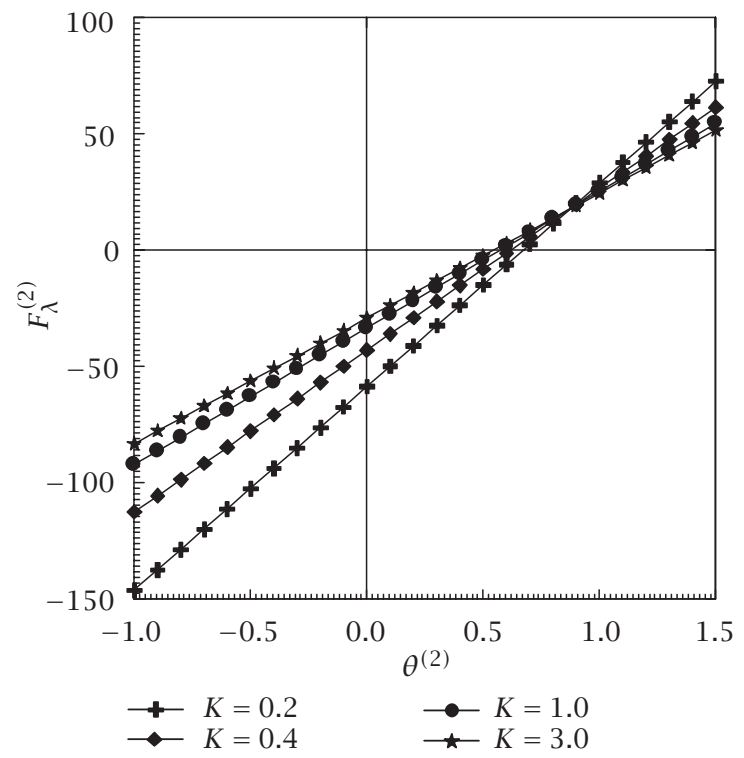

(b) The frictionless force per wavelength $F_{\lambda}^{(2)}$ versus the dimensionless flow rate $\theta^{(2)}$ for $\operatorname{Re}=1, \varphi=0.3, M=2$, $\delta=0.06$, and different values of $K$.

FIGURE 6.3 


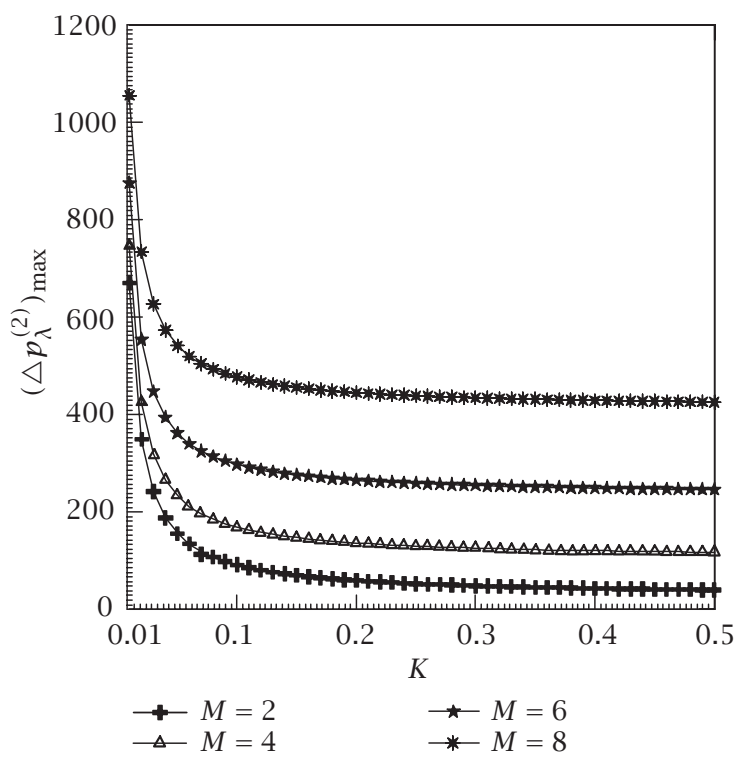

(a) The maximum pressure gradient per wavelength $\left(\triangle p_{\lambda}^{(2)}\right)_{\max }$ versus the permeability parameter $K$ for $\mathrm{Re}=$ $1, \varphi=0.2, \delta=0.06$, and different values of $M$.

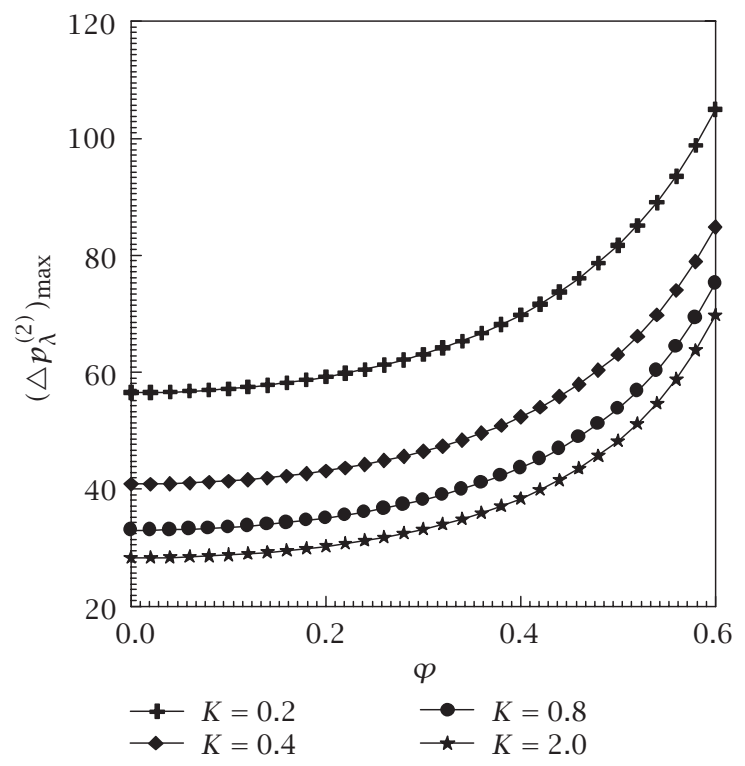

(b) The maximum pressure gradient per wavelength $\left(\triangle p_{\lambda}^{(2)}\right)_{\max }$ versus the amplitude ratio $\varphi$ for $\mathrm{Re}=1$, $M=2.5, \delta=0.06$, and different values of $K$.

FIGURE 6.4 


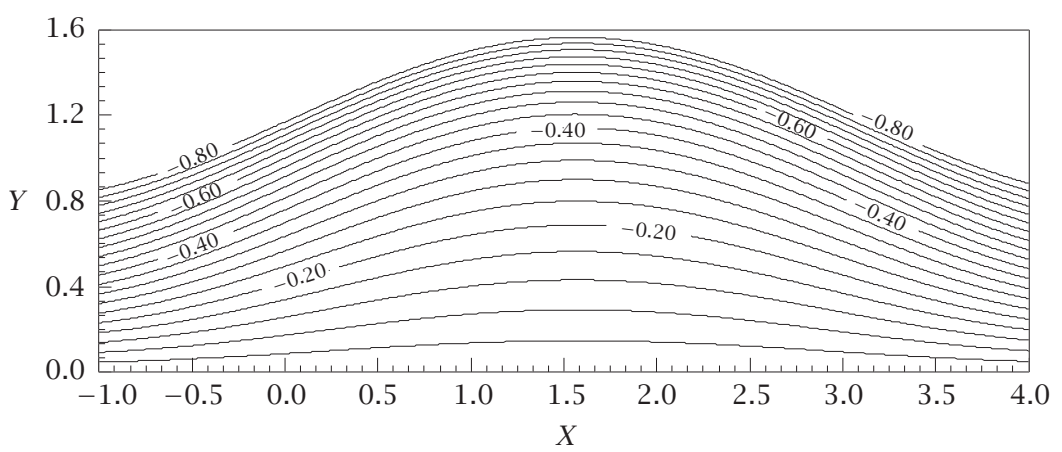

(a)

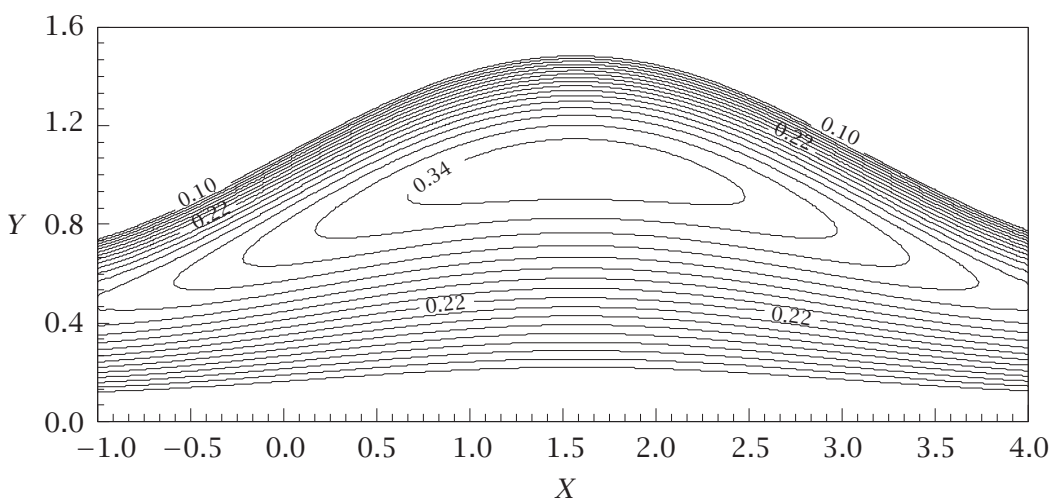

(b)

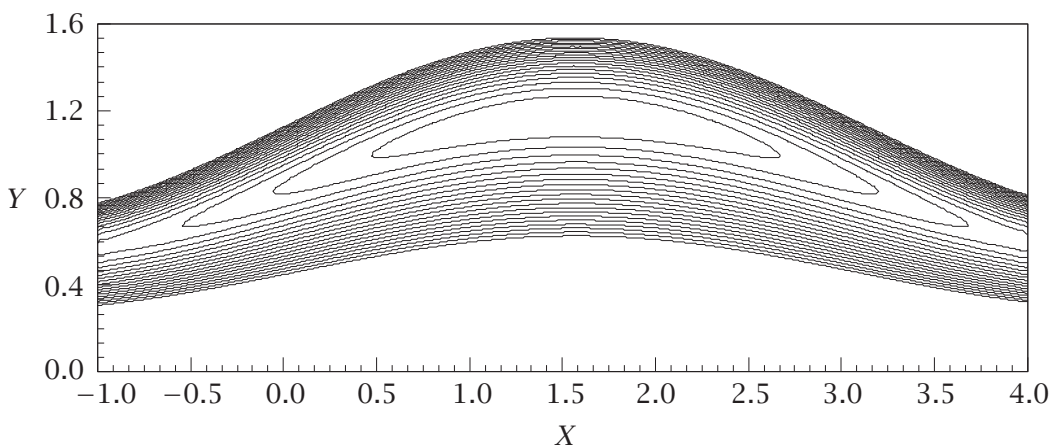

(c)

FIGURE 6.5. The streamlines for $M=1.5, \varphi=0.4, \delta=0.06, K=1.0$, and $\operatorname{Re}=0$ at (a) $\theta^{(2)}=0.4$, (b) $\theta^{(2)}=1.2$, and (c) $\theta^{(2)}=1.8$. 


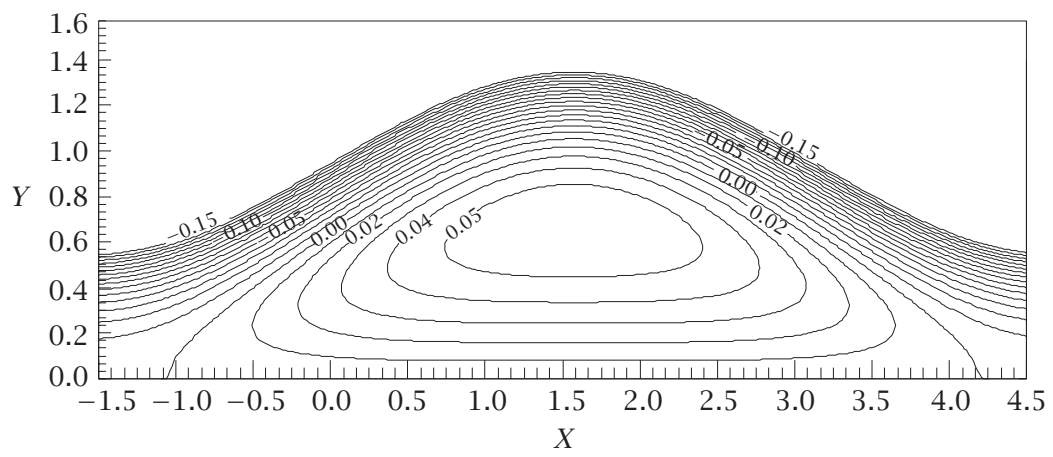

(a)

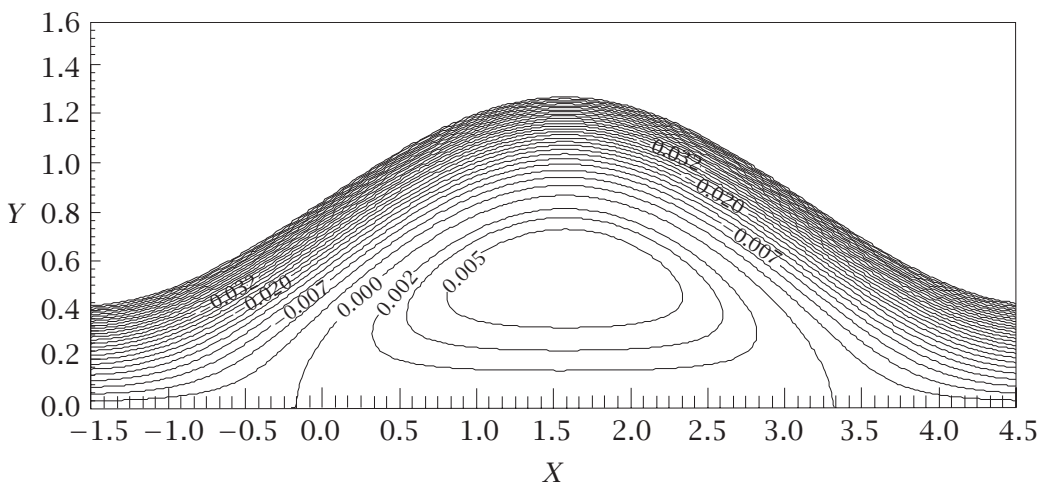

(b)

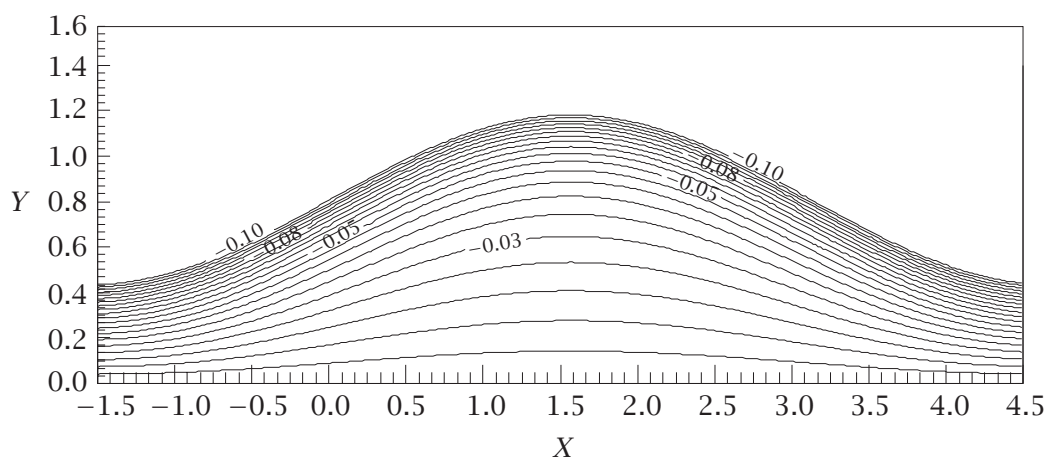

(c)

Figure 6.6. The streamlines for $K=1, \varphi=0.4, \delta=0.06, \theta^{(2)}=0.8$, and $\operatorname{Re}=1$, at (a) $M=2$, (b) $M=5$, and (c) $M=7$. 


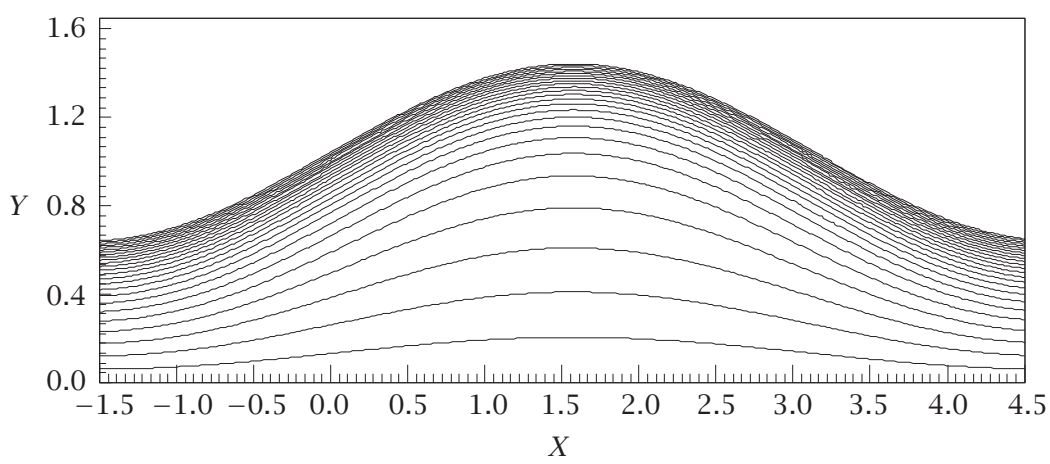

(a)

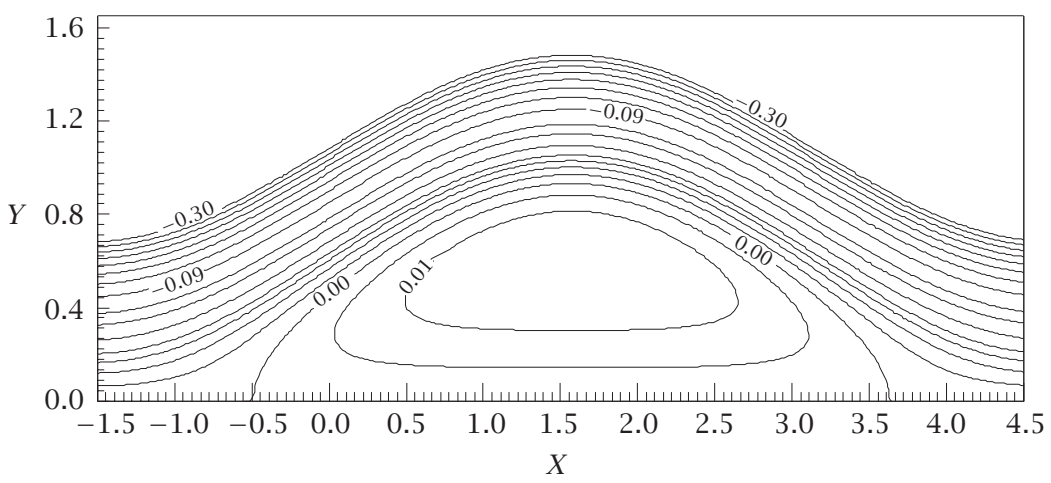

(b)

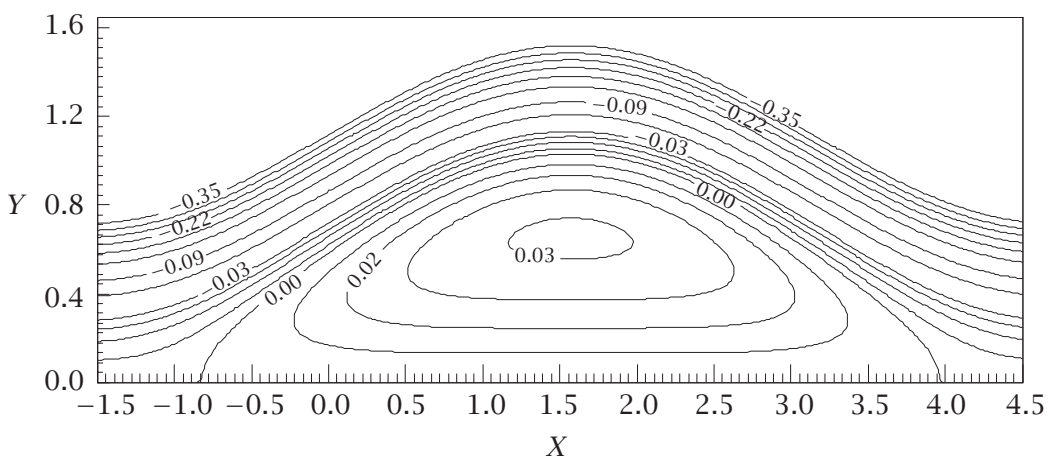

(c)

FIGURE 6.7. The streamlines for $M=2, \varphi=0.4, \delta=0.06, \theta^{(2)}=0.8$, and $\mathrm{Re}=1$, at (a) $K=0.02$, (b) $K=0.08$, and (c) $K=0.15$. 


\section{APPENDICES}

A. Constants of the first-order solution in $\delta$ in terms of $F^{(2)}$

$$
\begin{aligned}
c_{01}= & \frac{F^{(2)}+(1 / \gamma) \tanh \gamma h}{h-(1 / \gamma) \tanh \gamma h}, \quad c_{02}=\frac{c_{01}+1}{\cosh \gamma h}, \quad c_{03}=\gamma c_{01}^{\prime} c_{02}, \\
c_{04}= & -\left(c_{01} c_{02}^{\prime}+c_{01}^{\prime} c_{02}\right), \quad c_{11}=\frac{\operatorname{Re}}{4 \gamma^{2}} c_{03}, \quad c_{12}=\frac{\operatorname{Re}}{2 \gamma^{2}}\left(c_{04}-\frac{3}{2} c_{03}\right), \\
c_{13}= & \frac{3 \operatorname{Re}}{8 \gamma^{4}} c_{03}+\frac{c_{14}-h c_{15}}{\sinh \gamma h-\gamma h \cosh \gamma h}-\frac{\operatorname{Re}}{4 M^{3}} c_{04}, \\
c_{14}= & -c_{11} h^{2} \sinh \gamma h-c_{12} h \cosh \gamma h-\left(\frac{3 \operatorname{Re}}{8 \gamma^{4}} c_{03}+\frac{\operatorname{Re}}{4 M^{3}} c_{04}\right) \sinh \gamma h, \\
c_{15}= & -c_{11}\left(\gamma h^{2} \cosh \gamma h+2 h \sinh \gamma h\right)-c_{12}(\gamma h \sinh \gamma h+\cosh \gamma h) \\
& -\left(\frac{3 \operatorname{Re} c_{03}}{8 \gamma^{3}}+\frac{\operatorname{Re}}{4 \gamma^{2}} c_{04}\right) \cosh \gamma h, \quad D=\frac{\left(c_{14}-h c_{15}\right)}{(h-(1 / \gamma) \tanh \gamma h)}+c_{15} .
\end{aligned}
$$

B. Constants of the second-order solution in $\delta$ in terms of $F^{(2)}$

$$
E=-\frac{\left(h c_{42}-c_{41}\right)}{\gamma \cosh \gamma h(h-(1 / \gamma) \tanh \gamma h)}, \quad A=\gamma^{2}\left(c_{42}+\gamma E \cosh \gamma h\right),
$$

where,

$$
\begin{aligned}
c_{41}= & c_{34} h^{4} \sinh \gamma h+c_{35} h^{3} \cosh \gamma h+c_{36} h^{2} \sinh \gamma h+c_{37} h \cosh \gamma h \\
& +c_{38} \sinh \gamma h+c_{39} h \cosh 2 \gamma h+c_{40} \sinh 2 \gamma h-\frac{c_{29}}{\gamma^{2}}\left(h^{3}+\frac{6 h}{\gamma^{2}}\right), \\
c_{42}= & \gamma c_{34} h^{4} \cosh \gamma h+\left(4 c_{34}+\gamma c_{35}\right) h^{3} \sinh \gamma h \\
& +\left(3 c_{35}+\gamma c_{36}\right) h^{2} \cosh \gamma h+\left(2 c_{36}+\gamma c_{37}\right) h \sinh \gamma h+\left(c_{37}+\gamma c_{38}\right) \cosh \gamma h \\
& +2 \gamma h c_{39} \sinh 2 \gamma h+\left(c_{39}+2 \gamma c_{40}\right) \cosh 2 \gamma h-\frac{c_{29}}{\gamma^{2}}\left(3 h^{2}+\frac{6}{\gamma^{2}}\right), \\
c_{34}= & \frac{c_{27}}{8 \gamma}, \quad c_{35}=-\frac{c_{27}}{4 \gamma^{2}}+\frac{c_{28}}{6 \gamma}, \quad c_{36}=\frac{3 c_{27}}{8 \gamma^{3}}-\frac{c_{28}}{4 \gamma^{2}}+\frac{c_{31}}{4 \gamma}, \\
c_{37}= & \frac{3 c_{27}}{8 \gamma^{4}}+\frac{c_{28}}{4 \gamma^{3}}-\frac{c_{31}}{4 \gamma^{2}}+\frac{c_{33}}{2 \gamma}, \quad c_{38}=\frac{3 c_{27}}{16 \gamma^{5}}-\frac{c_{28}}{8 \gamma^{4}}+\frac{c_{31}}{8 \gamma^{3}}, \quad c_{39}=\frac{c_{30}}{3 \gamma^{2}}, \\
c_{40}= & -\frac{4 c_{30}}{9 \gamma^{3}}+\frac{c_{32}}{3 \gamma^{2}}, \quad c_{33}=-\frac{24 c_{26}}{\gamma^{5}}+\frac{6 c_{24}}{\gamma^{4}}-\frac{2 c_{25}}{\gamma^{3}}+\frac{c_{22}}{\gamma^{2}}+\frac{2 c_{02}^{\prime \prime}}{\gamma}, \\
c_{32}= & -\frac{\left(c_{20}+c_{23}\right)}{8 \gamma^{3}}+\frac{1}{8 \gamma^{2}} c_{21}, \quad c_{31}=\frac{18 c_{26}}{\gamma^{4}}-\frac{4 c_{24}}{\gamma^{3}}+\frac{c_{25}}{\gamma^{2}}, \\
c_{30}= & \frac{1}{8 \gamma^{2}}\left(c_{20}+c_{23}\right), \quad c_{29}=-\frac{1}{12}\left(c_{20}+c_{23}\right), \quad c_{28}=\frac{6 c_{26}}{\gamma^{3}}+\frac{1}{\gamma^{2}} c_{24},
\end{aligned}
$$


$c_{27}=\frac{1}{\gamma^{2}} c_{26}, \quad c_{26}=-\operatorname{Re} \gamma^{3} c_{01}^{\prime} c_{11}^{\prime}$,

$c_{25}=\operatorname{Re}\left(4 \gamma c_{01} c_{11}^{\prime}+\gamma^{2} c_{12}^{\prime} c_{01}-D^{\prime} c_{02}-6 c_{01}^{\prime} c_{11}-3 \gamma^{2} c_{01}^{\prime} c_{12}-\gamma^{3} c_{01}^{\prime} c_{13}\right)$,

$c_{24}=\operatorname{Re}\left(\gamma^{2} c_{01} c_{11}^{\prime}-6 \gamma^{2} c_{01}^{\prime} c_{11}-\gamma^{3} c_{01}^{\prime} c_{12}\right), \quad c_{23}=-4.0 \operatorname{Re} \gamma c_{02} c_{11}^{\prime}$,

$c_{22}=\operatorname{Re}\left(\frac{D}{\gamma} c_{02}^{\prime}+2 c_{01} c_{11}^{\prime}+2 \gamma c_{12}^{\prime} c_{01}+\gamma^{2} c_{01} c_{13}^{\prime}\right)$,

$c_{21}=\operatorname{Re}\left(6 c_{02}^{\prime} c_{11}-2 c_{02} c_{11}^{\prime}+2 \gamma c_{02} c_{12}^{\prime}+2 \gamma c_{02}^{\prime} c_{12}\right), \quad c_{20}=4 \operatorname{Re} \gamma c_{11} c_{02}^{\prime}$.

\section{REFERENCES}

[1] H. L. Agrawal and B. Anwaruddin, Peristaltic flow of blood in a branch, Ranchi Univ. Math. J. 15 (1984), 111-121.

[2] T. D. Brown and T.-K. Hung, Computational and experimental investigations of two-dimensional nonlinear peristaltic flows, J. Fluid Mech. 83 (1977), 249274.

[3] J. C. Burns and T. Parkes, Peristaltic motion, J. Fluid Mech. 29 (1967), 731-743.

[4] N. T. El-Dabe and S. M. El-Mohendis, Magnetohydrodynamic flow of a second order fluid through a porous medium on an inclined porous plate, Arabian J. Sci. Engrg. 20 (1995), 571.

[5] E. F. Elshehawey and Kh. S. Mekheimer, Couple-stresses in peristaltic transport of fluids, J. Phys. D: Appl. Phys. 27 (1994), 1163-1170.

[6] V. V. Prasad and N. C. P. Ramacharyulu, Unsteady flow of a dusty incompressible fluid between two parallel plates under an impulsive pressure gradient, Defence Sci. J. 30 (1981), 125-130.

[7] M. Y. Jaffrin, Inertia and streamline curvature effects on peristalsis pumping, Int. J. Engng. Sci. 11 (1973), 681-699.

[8] M. Y. Jaffrin and A. H. Shapiro, Peristaltic pumping, Ann. Rev. Fluid Dynamics 3 (1971), 13-36.

[9] M. Y. Jaffrin, A. H. Shapiro, and S. L. Weinberg, Peristaltic pumping with long wavelengths at low Reynolds number, J. Fluid Mech. 37 (1969), no. 4, 799825.

[10] T. W. Latham, Fluid motion in a peristaltic pump, Master's thesis, MIT, Massachusetts, 1966.

[11] Kh. S. Mekheimer, E. F. El Shehawey, and A. M. Elaw, Peristaltic motion of a particlefluid suspension in a planar channel, Int. J. Theor. Phys. 37 (1998), no. 11, 2895-2920.

[12] R. A. Ramachandra and S. Usha, Peristaltic transport of two immiscible viscous fluids in a circular tube, J. Fluid Mech. 298 (1995), 271-285.

[13] A. Rapits and C. Perdikis, Flow of a viscous fluid through a porous medium bounded by a vertical surface, Int. J. Eng. Sci. 27 (1983), 1327-1330.

[14] H. J. Rath, Peristaltische Stromungen, Springer-Verlag, Berlin, 1980.

[15] A. E. Scheidegger, The Physics of Flow through Porous Media, 3rd ed., University of Toronto Press, Toronto, Canada, 1974.

[16] A. M. Siddiqui and W. H. Schwarz, Peristaltic flow of a second order fluid in tubes, J. Non-Newtonian Fluid Mech. 35 (1994), 257-284.

[17] L. M. Srivastava and R. P. Agrawal, Oscillating flow of a conducting fluid with a suspension of spherical particles, J. Appl. Mech. 47 (1980), 196-199.

[18] L. M. Srivastava and V. P. Srivastava, Peristaltic transport of blood: Casson ModelII, J. Biomech. 17 (1984), 821-829. 
[19] S. Takabatake and K. Ayukawa, Numerical study of two-dimensional peristaltic flows, J. Fluid Mech. 122 (1982), 439-465.

[20] S. Takabatake, K. Ayukawa, and A. Mori, Peristaltic pumping in circular cylindrical tubes: a numerical study of fluid transport and its efficiency, J. Fluid Mech. 193 (1988), 267-283.

[21] D. Tong and M. Shen, Non-stationary peristaltic and transport of a heatconducting fluid, J. Math. Anal. Appl. 174 (1993), no. 1, 265-289.

[22] C. L. Varshney, The fluctuating flow of a viscous fluid through a porous medium bounded by a porous and a horizontal surface, Indian J. Pure Appl. Math. 10 (1979), 1558.

[23] T. F. Zien and S. A. strach, Long wave approximation to peristaltic motion, J. Biomech. 3 (1970), 63-75.

Kh. S. Mekheimer: Department of Mathematics, Faculty of Science, Al-Azhar University, Nasr City, 11884, Cairo, Egypt

E-mail address: kh_mekheimer@yahoo.com

T. H. Al-Arabi: Girls College, Madina Munawwarah 3193, Saudi Arabia 


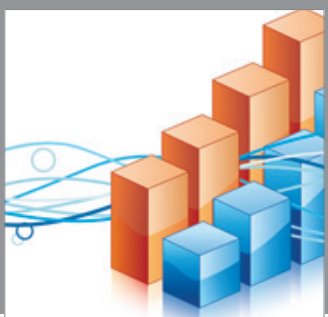

Advances in

Operations Research

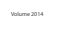

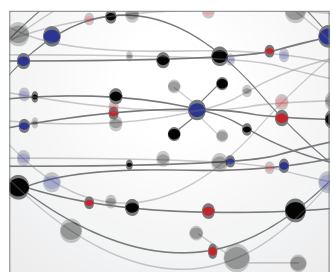

\section{The Scientific} World Journal
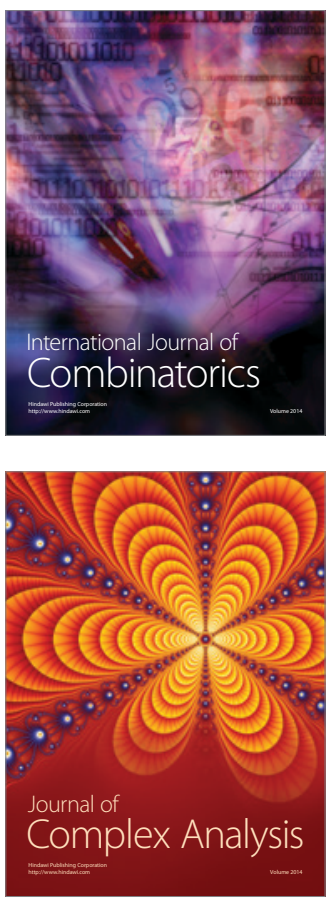

International Journal of

Mathematics and

Mathematical

Sciences
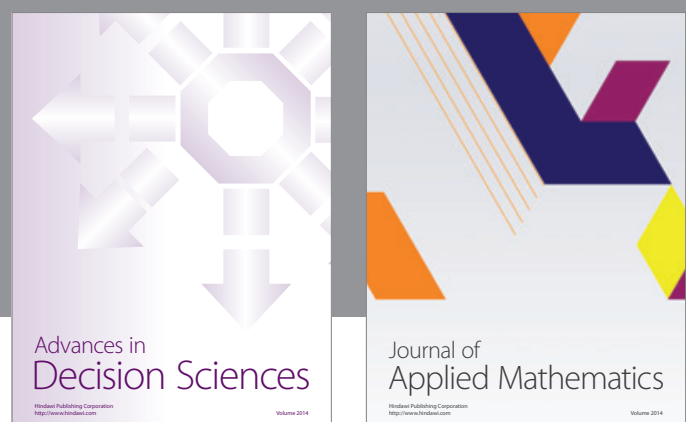

Journal of

Applied Mathematics
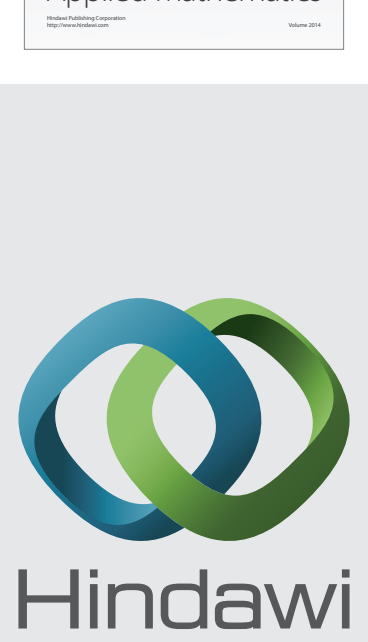

Submit your manuscripts at http://www.hindawi.com
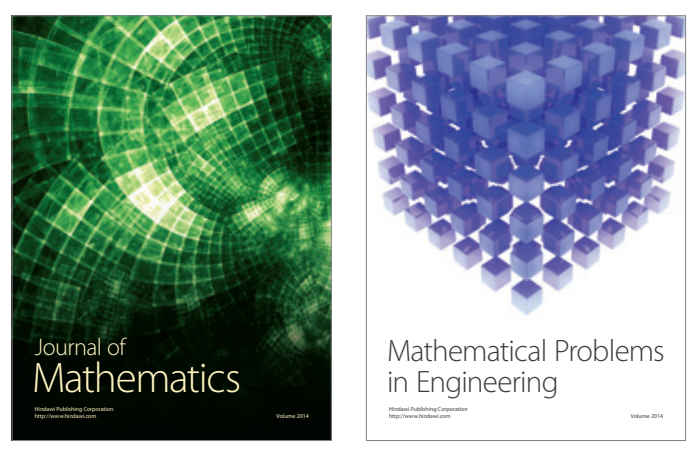

Mathematical Problems in Engineering
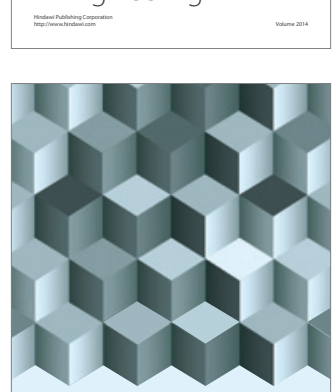

Journal of

Function Spaces
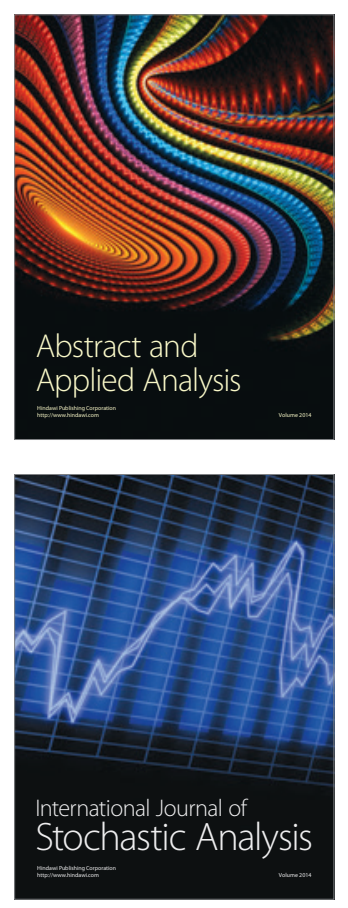

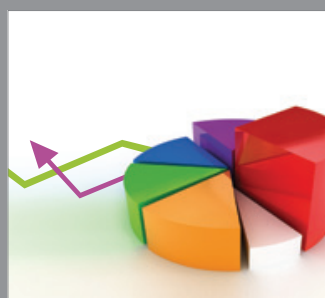

ournal of

Probability and Statistics

Promensencen
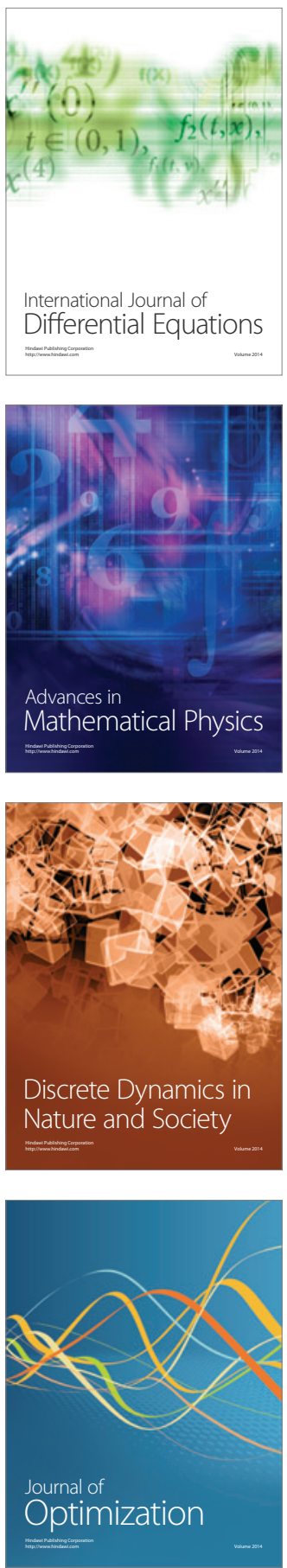